\title{
Successful laparoscopic conversion surgery for gastric cancer with para-aortic lymph node metastasis after third-line chemotherapy: a case report
}

\author{
Yuki Ushimaru ${ }^{1} \oplus$ - Kazuhiro Nishikawa ${ }^{1} \cdot$ Yumiko Yasuhara $^{2} \cdot$ Ryohei Kawabata $^{1} \cdot$ Yoichi Makari $^{1}$. Junya Fujita ${ }^{1}$. \\ Akihiro Kitagawa ${ }^{1} \cdot$ Nobuyoshi Ohara $^{1} \cdot$ Yuichiro Miyake ${ }^{1} \cdot$ Sakae Maeda $^{1} \cdot$ Shin Nakahira ${ }^{1} \cdot$ Ken Nakata $^{1}$. \\ Atsushi Miyamoto ${ }^{1}$
}

Received: 25 June 2021 / Accepted: 9 October 2021 / Published online: 13 October 2021

(c) The Japan Society of Clinical Oncology 2021

\begin{abstract}
We herein reported a case of advanced gastric cancer (GC) with para-aortic lymph node (PALN) metastases who successful achieved downstaging following systemic chemotherapy and underwent curative laparoscopic conversion surgery. A 74-year-old male patient diagnosed with advanced GC and PALN metastases [cT4N3M1(LYM), stage IVA] was administered chemotherapy and immunotherapy for 28 months. After 27 courses of nivolumab as third-line chemotherapy, PALN enlargement was resolved, for which conversion surgery was planned. Subsequently, laparoscopic distal D2 gastrectomy with sampling para-aortic lymphadenectomy was performed, after which a pathological diagnosis of type V moderately differentiated tubular adenocarcinoma with mucinous adenocarcinoma, stage ypT3 (SS), ly1c, and v0, was established. The pathological proximal and distal tumor margins were negative. One lymph node metastasis was observed (No. 6; 1/25). The sampled lymph nodes were negative (No. 16a1: 0/2). The therapeutic effect was categorized as Grade 1a. The postoperative course was uneventful, with the patient receiving nivolumab to control for potential PALN metastases. Postoperatively, no recurrence was observed over 11 months. Laparoscopic conversion gastrectomy was successfully performed in a patient with advanced GC that was originally unresectable, suggesting that minimally invasive surgery may be a good option for originally unresectable advanced GC that becomes resectable.
\end{abstract}

Keywords Gastric cancer · Conversion surgery $\cdot$ Laparoscopic surgery $\cdot$ Immune checkpoint inhibitor

\section{Introduction}

Gastric cancer (GC) is the second most common cause of cancer death worldwide [1]. As the world confronts the coronavirus disease 2019 (COVID-19) pandemic, reports regarding the deprioritization, delay, and discontinuation of cancer have emerged [2]. Moreover, the diagnosis of new patients with GC has also decreased, indicating that the decrease in the number of gastrointestinal endoscopies has certainly affected the detection and progression of GC [3].

Yuki Ushimaru

ushimaru-y@sakai-hospital.jp

1 Department of Surgery, Sakai City Medical Center, 1-1-1 Ebaraji-cho, Nishi-ku, Sakai City, Osaka, Japan

2 Department of Pathology, Sakai City Medical Center, Sakai City, Osaka, Japan
These circumstances would facilitate a future in which more patients present with advanced GC than ever before, subsequently requiring multimodal cancer treatments.

Conversion therapy is defined as a surgical treatment aimed at R0 resection after chemotherapy for tumors that, for technical and/or oncological reasons, were originally unresectable [4]. Despite the availability of standardized criteria for unresectable and metastatic cancers, assessment of resectability after chemotherapy and timing of surgery have yet to be standardized. Studies have clearly shown that R0 surgery after induction chemotherapy with partial or complete response appears to provide better survival outcomes compared to chemotherapy alone [5-7]. The results of the ATTRACTION-2 study showed that nivolumab as third- or later-line chemotherapy prolongs overall survival in patients with advanced GC $[8,9]$. However, the clinical indication and prognostic significance of conversion therapy remain unclear in responders to nivolumab. 
Minimally invasive surgery (MIS), such as laparoscopic and robotic gastrectomy, has been widely considered the optimal approach for early GC [10-12]. Recent evidence has suggested that patients with advanced GC can benefit from MIS, which showed postoperative complications and longterm outcomes comparable to those for conventional open surgery [13-15]. MIS may also reduce deaths from other diseases, such as pneumonia [16], and may be indicated for elderly patients with advanced GC.

We herein presented a case of laparoscopic conversion surgery for GC with multiple para-aortic lymph node (PALN) metastases who received nivolumab as third-line chemotherapy.

\section{Case report}

A 74-year-old male with abdominal distension was admitted to our department with advanced GC. Preoperative esophagogastroduodenoscopy revealed a circumferential type 3 tumor extending from the middle to lower portions of the gastric body (Fig. 1a). Biopsy revealed moderately to poorly differentiated adenocarcinoma (Fig. 1b, c) with positive human epidermal growth factor receptor type 2 (HER2) upon immunohistochemical staining. Subsequent preoperative computed tomography $(\mathrm{CT})$ revealed gastric wall thickening at the primary tumor site without surrounding organs invasion (Fig. 2a) and enlarged regional and PALNs (No. 16b1) (Fig. 2b); the clinical stage was cT4aN2M1(LYM) [17]. The patient received capecitabine, cisplatin, and trastuzumab (XPH) as first-line chemotherapy (XPH at $8 \mathrm{mg} / \mathrm{kg}$ on day 1 for the first cycle and then $6 \mathrm{mg}$ / $\mathrm{kg}$ for subsequent cycles, oral capecitabine at $3000 \mathrm{mg} / \mathrm{m}^{2} /$ day on days $1-14$, and cisplatin at $80 \mathrm{mg} / \mathrm{m}^{2}$ on day 1 , every 3 weeks). After three cycles, CT revealed regression of the primary tumor and regional and PALNs. After ten cycles of XPH, the primary tumor and another PALNs (No. 16a1) had increased in size. After five cycles of paclitaxel plus ramucirumab (paclitaxel at $80 \mathrm{mg} / \mathrm{m}^{2}$ and ramucirumab at $8 \mathrm{mg} / \mathrm{kg}$ ) as second-line chemotherapy, the primary tumor still remained (Fig. 2d), whereas PALNs (No. 16a1) had increased in size (Fig. 2e, f). Therefore, third-line chemotherapy with nivolumab at $240 \mathrm{mg} / \mathrm{body}$ was initiated. After six cycles of nivolumab, a notable reduction in the size of PALNs (No. 16b1) was observed with mild reduction of the primary tumor site.

Given the absence of immune-related adverse effects, nivolumab was continued for 27 courses, with subsequent endoscopy and follow-up CT revealing no changes in the primary tumor (Fig. 1d, 2g). Biopsy revealed moderately to
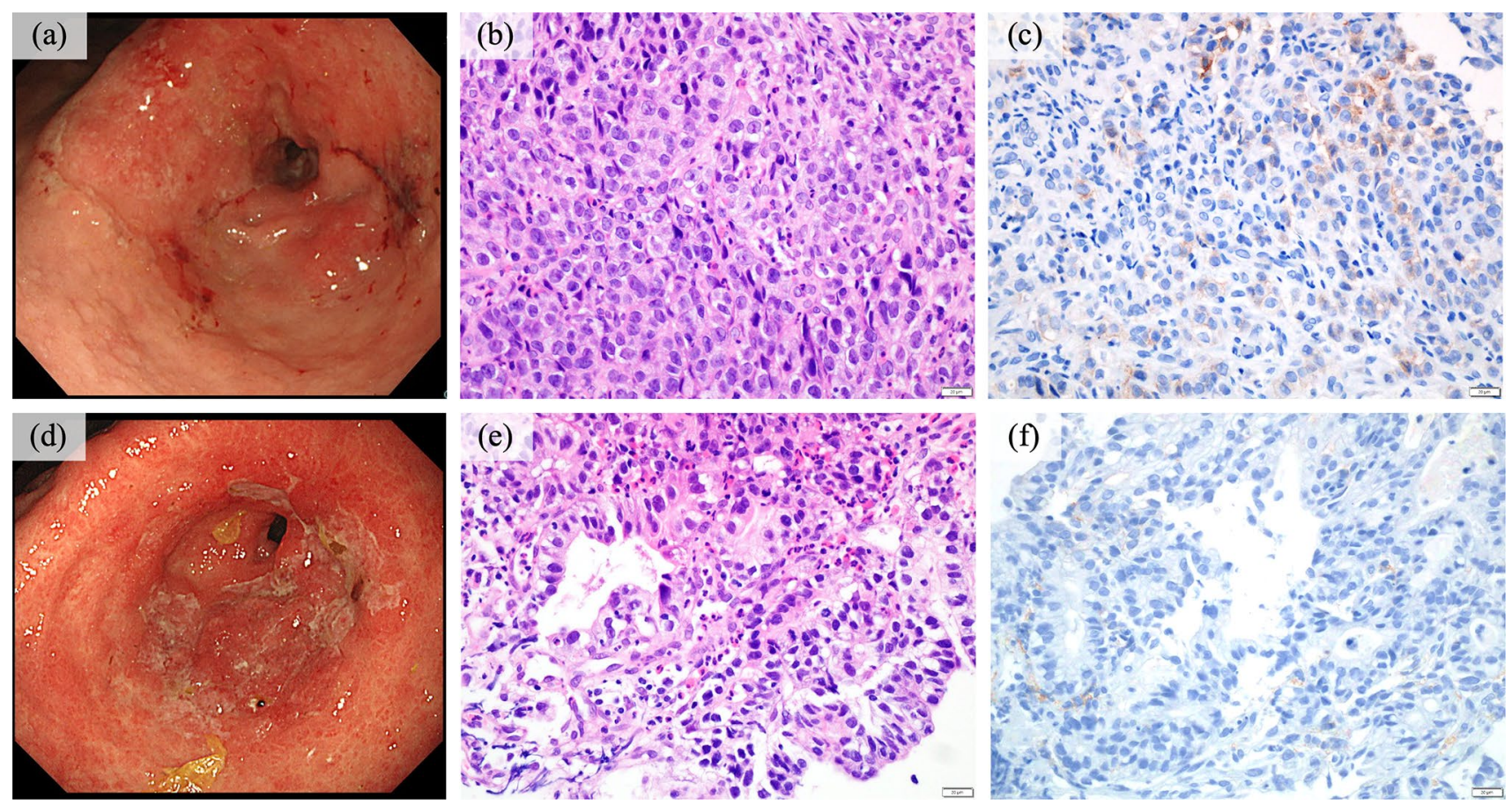

Fig. 1 Endoscopic and pathological findings. Upper panel: upon initial visit. Type 3 advanced gastric cancer was identified occupying the entire circumference of the lower third region of the stomach (a). Pathological examination revealed a moderately to poorly differentiated tubular adenocarcinoma (b) and PD-L1(22C3) positivity (c). Lower panel: During the follow-up examination after third-line chemotherapy. The primary lesion was mildly reduced but stenotic (d). Pathological examination indicated adenocarcinoma (e), although negative for PD-L1(22C3) (f) 

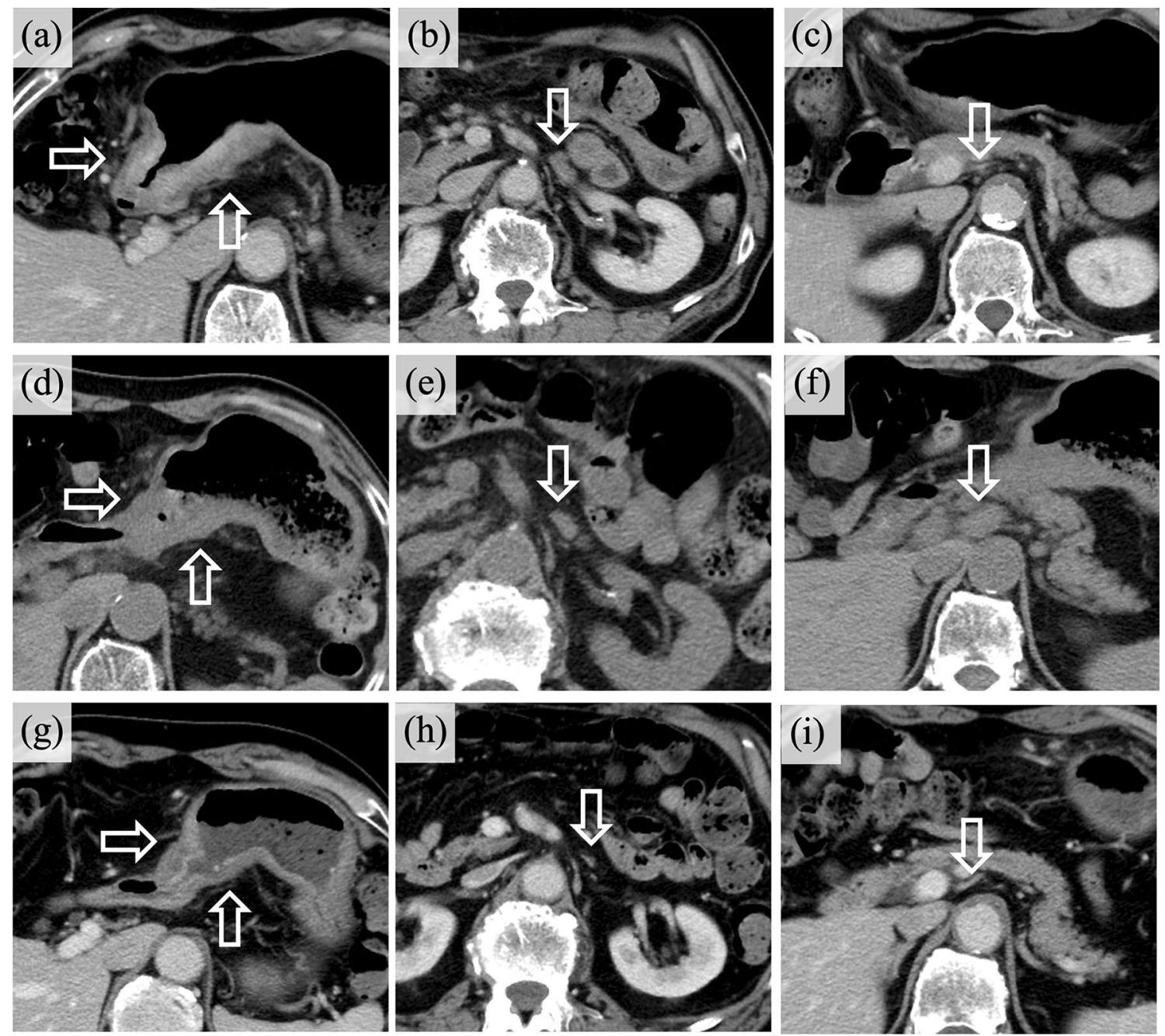

Fig. 2 Computed tomography findings. Upper panel: first computed tomography (CT) image showing gastric wall thickening at the tumor site (white arrows) (a). Para-aortic lymph nodes (PALN) were enlarged (16b1, white arrow) (b). Initially, no enlarged PALNs were observed in 16a1 (white arrow) (c). Middle panel: CT images before nivolumab therapy showing decreased gastric wall thickness in

poorly differentiated adenocarcinoma (Fig. 1e, f). Followup CT revealed a decrease in the size of the regional and PALNs (Fig. 2h, i), whereas fluorodeoxyglucose positron emission tomography showed a negative response in PALNs; the clinical stage was ycT3N2M0. Considering the aforementioned findings, we decided to perform laparoscopic gastrectomy given the prospect of radical resection and the onset of stenosis symptoms caused by the primary lesion. Throughout the surgery, we found no intraperitoneal nodules indicating metastasis, whereas cytology results came back negative. Moreover, given that the frozen sections were negative for PALN, laparoscopic D2 distal gastrectomy was performed. The total operative time was $5 \mathrm{~h}$ and $18 \mathrm{~min}$, with a total blood loss of $80 \mathrm{~mL}$, forgoing the need for transfusions. Postoperative delirium was observed the day after the primary tumor site (white arrows) (d). Although a PALN (16b1) had decreased in size (white arrow) (e), another PALN (16a1) was enlarged (white arrow) (f). Lower panel: CT images after nivolumab therapy showing no changes in gastric wall thickness at the primary lesion (white arrows) (g). The CT image showed complete response by PALN (white arrow) $(\mathbf{h}, \mathbf{i})$

the surgery, with the patient needing a few days to wean. Finally, he was allowed to leave the hospital on the 12th day after the surgery.

The pathological findings of the postoperative excised specimens were as follows. Macroscopically, the tumor measured $90 \times 65 \mathrm{~mm}$ and was classified as type 5 , with a scar-like appearance at the center of the tumor. Histological findings suggested moderately to poorly differentiated tubular adenocarcinoma with focally mucinous adenocarcinoma component, stage ypT3 (SS), Ly1c, and V0 (Fig. 3). The therapeutic effect was categorized as Grade 1a. HER2 immunohistochemical staining, which was positive before chemotherapy, returned negative. The pathological proximal and distal margins were free from carcinoma cells. Although seven metastatic lymph nodes were observed, 


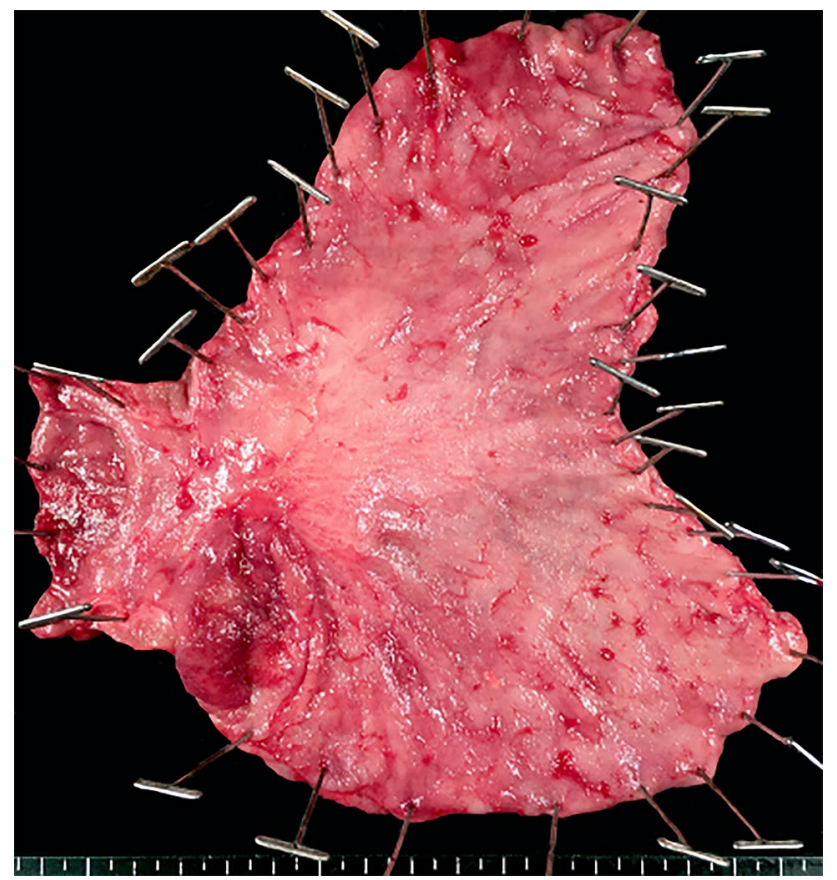

Fig. 3 Macroscopic findings of the resected stomach. Mucosal side of the stomach. The tumor classified as type 5 and occupied the entire circumference of the lower third region of the stomach

viable cells were found in only one metastatic lymph node (No. 6: 1/25). Moreover, one of the two sampled lymph nodes showed metastasis but no viable cells (No. 16a1: 0/2). Immunohistochemical staining for CD4, CD8, CD68, and CD163 was then performed at three sites of the resected specimen: primary GC lesion (Fig. 4a, lymph node No. 6 with viable tumor cells (Fig. 4b), and lymph node No. 16a1 without viable tumor cells (Fig. 4c). In the primary lesion and metastatic residual lymph node, the panel showed negative staining and low macrophage density. On the other hand, the panel showed positive staining for CD8-dominant lymphocyte profile and high CD163/CD68 positive macrophage density in the lymph nodes that seemed to have become negative.

Currently, the patient is receiving nivolumab to control potential PALN metastases and has survived for 41 months after first-line chemotherapy, with no signs of disease recurrence 14 months after surgery.

\section{Discussion}

Generally, GC with non-curative factors carries a poor prognosis, with a median overall survival of approximately 13-16 months, even with standard chemotherapy [18-20]. The REGATTA trials showed that additional gastrectomy over chemotherapy alone provided no survival benefits in patients with non-curable advanced GC [21]. On the other hand, studies on unresectable advanced GC have shown that $\mathrm{R} 0$ resection after induction chemotherapy with partial or (a)

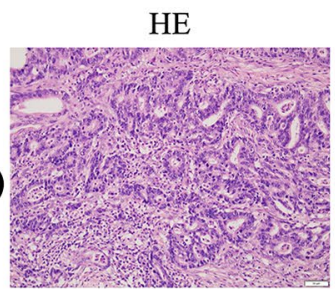

(b)

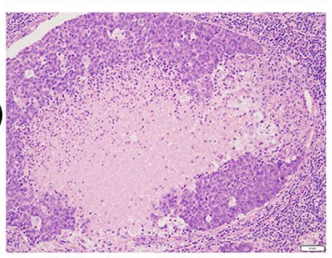

(c)

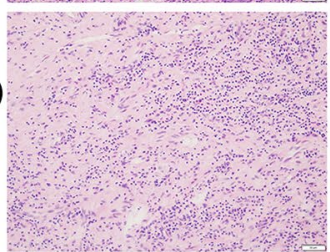

CD4
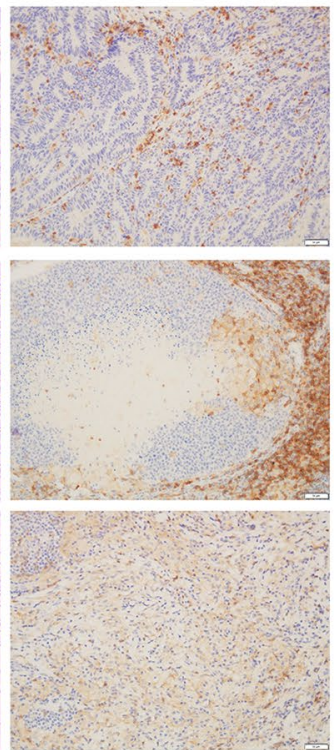

CD8
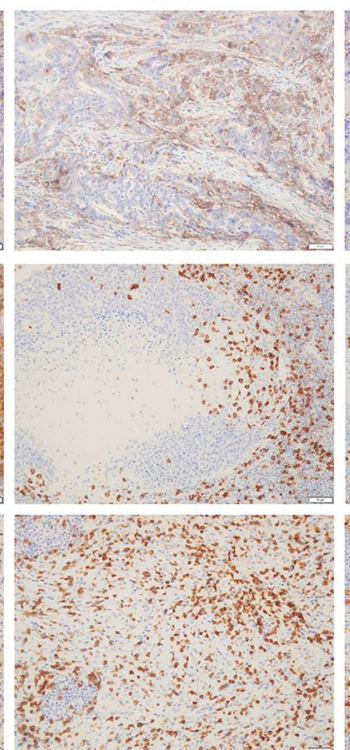

CD68
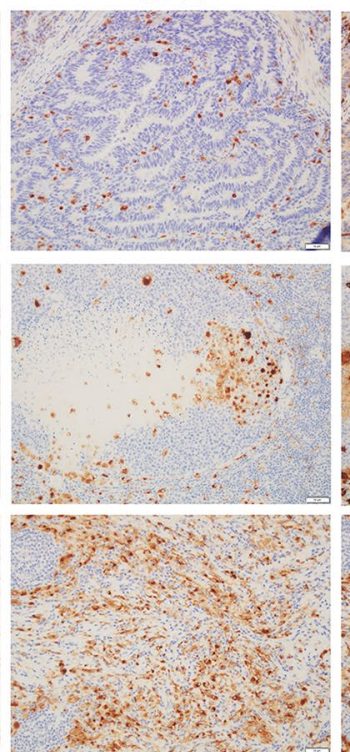
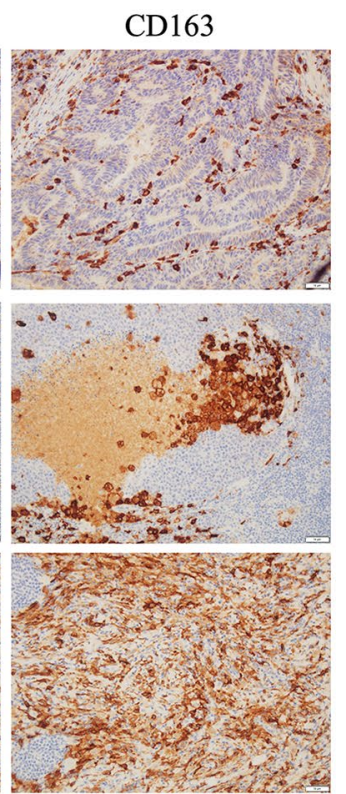

Fig. 4 Immunohistochemistry results. Upper panel: (a) Primary gastric cancer lesion. No lymphocyte infiltration was noted. Middle panel: (b) Lymph node with viable tumor cells (No. 6). The center of the lymph node was necrotic, and CD8-positive lymphocytes infil- trated from the failed area. Lower panel: (c) Lymph node showing a complete response (No. 16a1). Mainly CD8-positive lymphocytes infiltrated the stroma site, with some CD68-positive lymphocytes also being present 
complete response appears to provide better survival outcomes compared to chemotherapy alone [5-7]. Therefore, conversion gastrectomy has become an attractive option for stage IV GC with controlled distant metastasis. In the current case, the PALN metastasis showed a complete response to chemotherapy. Considering the findings of a previous study, which showed that prophylactic D2 dissection with extended lymphadenectomy did not improve the prognosis of curable GC [22], we opted to perform laparoscopic D2 distal gastrectomy with sampling para-aortic lymphadenectomy. Nonetheless, para-aortic lymphadenectomy during conversion surgery has remained controversial $[23,24]$ and requires additional investigations.

Laparoscopic gastrectomy, which is considered more effective than conventional open surgery, has been more commonly used for early GC given the recent technical and instrumental advancements [10-12]. Notably, a randomized phase II study (JLSSG0901) found that laparoscopic distal gastrectomy was technically safe for locally advanced GC [13], while another large phase III trial (KLASS-02-RCT) showed fewer complications, less pain, and faster recovery with laparoscopic distal gastrectomy [14]. Furthermore, a large phase III randomized clinical trial (CLASS-01) showed similar long-term outcomes [15]. Recent evidence has shown that MIS can be safely performed as conversion surgery following chemotherapy for stage IV GC [25], indicating the safety of MIS for conversion surgery, especially when no additional resection is planned.

In our case, the therapeutic effect had been categorized as Grade 1a in the primary lesion. However, the clinical course showed downstaging, that is, a reduction in the primary tumor volume and a complete response by the PALNs. Moreover, the Programmed Death Ligand 1 Immunohistochemistry Assay, which evaluates the effect of nivolumab, revealed a discrepancy between residual metastatic lymph nodes and negative lymph nodes - a result that seems to be exciting. The reasons for this are discussed below. Accordingly, pathological examination revealed that CD68-possitive macrophages and CD8 positive lymphocytes and macrophages infiltrated the lymph nodes that showed a complete response (Fig. 4C). On the other hand, the same phenomenon did not occur at the residual tumor area (Fig. 4A,B). The CD68-positive macrophages were also positive for CD163 (M2-like macrophage), a marker for immunosuppressive tumor-associated macrophages (TAMs). Considering the availability of similar reports in the past [26], the excellent therapeutic effects observed in our case can be attributed to the following scenarios: (1) TAMs suppressed CD8-positive lymphocytes, (2) nivolumab inhibited the negative interaction of M2 macrophages, and (3) CD8-positive lymphocytes were released and attacked the tumor. However, given that this is a case report, future case series are needed to validate such mechanisms.
There are still little data on postoperative adjuvant chemotherapy for stage IV GC that has reached conversion surgery. Despite a generally worse clinical status in stage IV GC, some previous study indicated that postoperative chemotherapy does have a survival benefit for GC patients with surgical resection that leaved no macroscopically visible disease $[27,28]$. The magnitude of the additive effect of postoperative adjuvant chemotherapy on prognosis is unknown, but the improvement in cure rate is evident. In this case, we performed a pick-up dissection of PALN in addition to the standard lymph nodes dissection. Also, there was a possibility that cancer was left in the remaining PALN. Therefore, we aimed to achieve complete cancer control by adding postoperative adjuvant chemotherapy. In the choice of drug, we chose nivolumab because of its high efficacy before surgery.

In conclusion, we herein reported a case of successful laparoscopic conversion surgery for GC with PALN metastases after third-line chemotherapy with nivolumab. Our results suggest that conversion surgery might be clinically beneficial for preventing tumor progression among responders to immune checkpoint inhibitors, particularly nivolumab therapy.

Acknowledgements There is no further acknowledgement that need to be made either to persons or institutions.

Funding The authors have not been funded for this work.

\section{Declarations}

Conflict of interest The authors have no competing interests to declare.

Ethical approval Not applicable.

Consent to participate Not applicable.

Consent for publication The patient provided written informed consent for the publication of this manuscript and images.

\section{References}

1. Ferlay J, Soerjomataram I, Dikshit R, Eser S, Mathers C, Rebelo M, Parkin DM, Forman D, Bray F (2015) Cancer incidence and mortality worldwide: sources, methods and major patterns in GLOBOCAN 2012. Int J Cancer 136(5):E359-386. https://doi. org/10.1002/ijc. 29210

2. The Lancet $O$ (2020) Safeguarding cancer care in a postCOVID-19 world. Lancet Oncol 21(5):603. https://doi.org/10. 1016/s1470-2045(20)30243-6

3. Lui TKL, Leung K, Guo CG, Tsui VWM, Wu JT, Leung WK (2020) Impacts of the coronavirus 2019 pandemic on gastrointestinal endoscopy volume and diagnosis of gastric and colorectal cancers: a population-based study. Gastroenterology 159(3):11641166.e1163. https://doi.org/10.1053/j.gastro.2020.05.037 
4. Yoshida K, Yamaguchi K, Okumura N, Tanahashi T, Kodera $\mathrm{Y}$ (2016) Is conversion therapy possible in stage IV gastric cancer: the proposal of new biological categories of classification. Gastric Cancer 19(2):329-338. https://doi.org/10.1007/ s10120-015-0575-z

5. Fukuchi M, Ishiguro T, Ogata K, Suzuki O, Kumagai Y, Ishibashi K, Ishida H, Kuwano H, Mochiki E (2015) Prognostic role of conversion surgery for unresectable gastric cancer. Ann Surg Oncol 22(11):3618-3624. https://doi.org/10.1245/s10434-015-4422-6

6. Morgagni P, Solaini L, Framarini M, Vittimberga G, Gardini A, Tringali D, Valgiusti M, Monti M, Ercolani G (2018) Conversion surgery for gastric cancer: a cohort study from a western center. Int J Surg 53:360-365. https://doi.org/10.1016/j.ijsu.2018.04.016

7. Zurleni T, Gjoni E, Altomare M, Rausei S (2018) Conversion surgery for gastric cancer patients: a review. World J Gastrointest Oncol 10(11):398-409. https://doi.org/10.4251/wjgo.v10.i11.398

8. Kang YK, Boku N, Satoh T, Ryu MH, Chao Y, Kato K, Chung HC, Chen JS, Muro K, Kang WK, Yeh KH, Yoshikawa T, Oh SC, Bai LY, Tamura T, Lee KW, Hamamoto Y, Kim JG, Chin K, Oh DY, Minashi K, Cho JY, Tsuda M, Chen LT (2017) Nivolumab in patients with advanced gastric or gastro-oesophageal junction cancer refractory to, or intolerant of, at least two previous chemotherapy regimens (ONO-4538-12, ATTRACTION-2): a randomised, double-blind, placebo-controlled, phase 3 trial. Lancet (London, England) 390(10111):2461-2471. https://doi.org/10.1016/s01406736(17)31827-5

9. Chen LT, Satoh T, Ryu MH, Chao Y, Kato K, Chung HC, Chen JS, Muro K, Kang WK, Yeh KH, Yoshikawa T, Oh SC, Bai LY, Tamura T, Lee KW, Hamamoto Y, Kim JG, Chin K, Oh DY, Minashi K, Cho JY, Tsuda M, Sameshima H, Kang YK, Boku $\mathrm{N}$ (2020) A phase 3 study of nivolumab in previously treated advanced gastric or gastroesophageal junction cancer (ATTRAC TION-2): 2-year update data. Gastric Cancer 23(3):510-519. https://doi.org/10.1007/s10120-019-01034-7

10. Katai H, Mizusawa J, Katayama H, Morita S, Yamada T, Bando E, Ito S, Takagi M, Takagane A, Teshima S, Koeda K, Nunobe S, Yoshikawa T, Terashima M, Sasako M (2020) Survival outcomes after laparoscopy-assisted distal gastrectomy versus open distal gastrectomy with nodal dissection for clinical stage IA or IB gastric cancer (JCOG0912): a multicentre, non-inferiority, phase 3 randomised controlled trial. Lancet Gastroenterol Hepatol 5(2):142-151. https://doi.org/10.1016/s2468-1253(19)30332-2

11. Kim W, Kim HH, Han SU, Kim MC, Hyung WJ, Ryu SW, Cho GS, Kim CY, Yang HK, Park DJ, Song KY, Lee SI, Ryu SY, Lee JH, Lee HJ (2016) Decreased morbidity of laparoscopic distal gastrectomy compared with open distal gastrectomy for stage i gastric cancer: short-term outcomes from a multicenter randomized controlled trial (KLASS-01). Ann Surg 263(1):28-35. https://doi.org/ 10.1097/sla.0000000000001346

12. Zeng YK, Yang ZL, Peng JS, Lin HS, Cai L (2012) Laparoscopyassisted versus open distal gastrectomy for early gastric cancer: evidence from randomized and nonrandomized clinical trials. Ann Surg 256(1):39-52. https://doi.org/10.1097/SLA.0b013e3182 $583 \mathrm{e} 2 \mathrm{e}$

13. Inaki N, Etoh T, Ohyama T, Uchiyama K, Katada N, Koeda K, Yoshida K, Takagane A, Kojima K, Sakuramoto S, Shiraishi N, Kitano S (2015) A Multi-institutional, prospective, phase II feasibility study of laparoscopy-assisted distal gastrectomy with D2 lymph node dissection for locally advanced gastric cancer (JLSSG0901). World J Surg 39(11):2734-2741. https://doi.org/ 10.1007/s00268-015-3160-z

14. Lee HJ, Hyung WJ, Yang HK, Han SU, Park YK, An JY, Kim W, Kim HI, Kim HH, Ryu SW, Hur H, Kong SH, Cho GS, Kim JJ, Park DJ, Ryu KW, Kim YW, Kim JW, Lee JH, Kim MC (2019) Short-term outcomes of a multicenter randomized controlled trial comparing laparoscopic distal gastrectomy with D2 lymphadenectomy to open distal gastrectomy for locally advanced gastric cancer (KLASS-02-RCT). Ann Surg 270(6):983-991. https://doi.org/10.1097/sla.0000000000003217

15. Yu J, Huang C, Sun Y, Su X, Cao H, Hu J, Wang K, Suo J, Tao K, He X, Wei H, Ying M, Hu W, Du X, Hu Y, Liu H, Zheng C, Li P, Xie J, Liu F, Li Z, Zhao G, Yang K, Liu C, Li H, Chen P, Ji J, Li G (2019) Effect of laparoscopic vs open distal gastrectomy on 3-year disease-free survival in patients with locally advanced gastric cancer: the CLASS-01 randomized clinical trial. JAMA 321(20):1983-1992. https://doi.org/10.1001/jama.2019.5359

16. Ushimaru Y, Kurokawa Y, Takahashi T, Saito T, Yamashita K, Tanaka K, Makino T, Yamasaki M, Nakajima K, Mori M, Doki Y (2020) Is laparoscopic gastrectomy more advantageous for elderly patients than for young patients with resectable advanced gastric cancer? World J Surg 44(7):2332-2339. https://doi.org/10.1007/ s00268-020-05486-2

17. A Japanese Gastric Cancer (2011) Japanese classification of gastric carcinoma 3rd English edition. Gastric Cancer 14(2):101-112. https://doi.org/10.1007/s10120-011-0041-5

18. Brenner H, Rothenbacher D, Arndt V (2009) Epidemiology of stomach cancer. Methods Mol Biol (Clifton, NJ) 472:467-477. https://doi.org/10.1007/978-1-60327-492-0_23

19. Liu X, Cai H, Sheng W, Wang Y (2012) Long-term results and prognostic factors of gastric cancer patients with microscopic peritoneal carcinomatosis. PLoS ONE 7(5):e37284. https://doi. org/10.1371/journal.pone.0037284

20. Yang D, Hendifar A, Lenz C, Togawa K, Lenz F, Lurje G, Pohl A, Winder T, Ning Y, Groshen S, Lenz HJ (2011) Survival of metastatic gastric cancer: significance of age, sex and race/ethnicity. J Gastrointest Oncol 2(2):77-84. https://doi.org/10.3978/j.issn. 2078-6891.2010.025

21. Fujitani K, Yang HK, Mizusawa J, Kim YW, Terashima M, Han SU, Iwasaki Y, Hyung WJ, Takagane A, Park DJ, Yoshikawa T, Hahn S, Nakamura K, Park CH, Kurokawa Y, Bang YJ, Park BJ, Sasako M, Tsujinaka T (2016) Gastrectomy plus chemotherapy versus chemotherapy alone for advanced gastric cancer with a single non-curable factor (REGATTA): a phase 3, randomised controlled trial. Lancet Oncol 17(3):309-318. https://doi.org/10. 1016/s1470-2045(15)00553-7

22. Sasako M, Sano T, Yamamoto S, Kurokawa Y, Nashimoto A, Kurita A, Hiratsuka M, Tsujinaka T, Kinoshita T, Arai K, Yamamura Y, Okajima K (2008) D2 lymphadenectomy alone or with para-aortic nodal dissection for gastric cancer. N Engl J Med 359(5):453-462. https://doi.org/10.1056/NEJMoa0707035

23. Dong YP, Deng JY (2020) Advances in para-aortic nodal dissection in gastric cancer surgery: a review of research progress over the last decade. World J Clin Cases 8(13):2703-2716. https://doi. org/10.12998/wjcc.v8.i13.2703

24. Kodera Y, Kobayashi D, Tanaka C, Fujiwara M (2015) Gastric adenocarcinoma with para-aortic lymph node metastasis: a borderline resectable cancer? Surg Today 45(9):1082-1090. https:// doi.org/10.1007/s00595-014-1067-1

25. Yamamoto K, Omori T, Hara H, Shinno N, Sugimura K, Miyata H, Takahashi H, Fujiwara Y, Ohue M, Yano M (2020) Minimally invasive surgery is feasible after preoperative chemotherapy for stage IV gastric cancer. Ann Gastroenterol Surg 4(4):396-404. https://doi.org/10.1002/ags3.12343

26. Watanabe H, Ohashi K, Nishii K, Seike K, Makimoto G, Hotta K, Maeda Y, Kiura K (2019) A long-term response to nivolumab in a case of PD-L1-negative lung adenocarcinoma with an EGFR mutation and surrounding PD-L1-positive tumor-associated Macrophages. Intern Med 58(20):3033-3037. https://doi.org/10.2169/ internalmedicine.2875-19

27. Yamaguchi T, Takashima A, Nagashima K, Makuuchi R, Aizawa M, Ohashi M, Tashiro K, Yamada T, Kinoshita T, Hata H, Kawachi Y, Kawabata R, Tsuji T, Hihara J, Sakamoto T, 
Fukagawa T, Katai H, Higuchi K, Boku N (2020) Efficacy of postoperative chemotherapy after resection that leaves no macroscopically visible disease of gastric cancer with positive peritoneal lavage cytology (CY1) or localized peritoneum metastasis (P1a): a multicenter retrospective study. Ann Surg Oncol 27(1):284-292. https://doi.org/10.1245/s10434-019-07697-x

28. Tiberio GA, Ministrini S, Gardini A, Marrelli D, Marchet A, Cipollari C, Graziosi L, Pedrazzani C, Baiocchi GL, La Barba G, Roviello F, Donini A, de Manzoni G (2016) Factors influencing survival after hepatectomy for metastases from gastric cancer. Eur J Surg Oncol 42(8):1229-1235. https://doi.org/10.1016/j.ejso. 2016.03.030

Publisher's Note Springer Nature remains neutral with regard to jurisdictional claims in published maps and institutional affiliations. 